Vol 13, Issue 6, 2020

\title{
TO STUDY PRESCRIPTION PATTERN IN THE MANAGEMENT OF OSTEOARTHRITIS IN TERTIARY CARE HOSPITAL
}

\author{
SHRIKANT B LAHAMATE*, SYED U RAZVI
}

Department of Pharmacology, Government Medical College, Aurangabad, Maharashtra, India. Email: shri.lahamate73@gmail.com Received: 04 February 2020, Revised and Accepted: 09 April 2020

\section{ABSTRACT}

Objective: The leading cause of disability is arthritis among adults worldwide. Osteoarthritis (OA) is the most common form of joint disease and the leading cause of pain in elderly people. The aim of the study was to study the prescription pattern in the management of OA.

Methods: A prospective observational study was conducted in a tertiary care hospital for period of 12 months in collaboration with the department of orthopedics. A total of 630 patients enrolled in the present study with fulfilling inclusion and exclusion criteria. Patients' data recorded in case report form and analyzed to study the prescription pattern.

Results: A total of 630 cases were enrolled in this study. Osteoarthritis is more common in female i.e. $55 \%$ followed by male i.e. $45 \%$ of patients. Old age (39.84\%) is most common risk factor followed by obesity (29.68\%) in OA patient. Most commonly prescribed drug was diclofenac followed by paracetamol. Nonsteroidal anti-inflammatory drugs were most commonly prescribed class of drug, i.e., $92.19 \%$. In this study, combination therapy most commonly prescribed, i.e., $75.4 \%$ followed by monotherapy, i.e., $24.6 \%$. Combination therapy in $0 A$ patients two drug therapy, i.e., $88.42 \%$ followed by three drug therapy, i.e., $10.53 \%$.

Conclusions: The principal aim of drug utilization research is to facilitate the rational use of drugs. The study shows that $\mathrm{OA}$ more common in female patient than male patient. The most common disease distribution site was knee in OA patient. Old age was the most common encounter risk factor in OA patient. Combination therapy was most commonly used than single drug therapy.

Keywords: Prescription pattern, Osteoarthritis, Nonsteroidal anti-inflammatory drugs, combination therapy.

(C) 2020 The Authors. Published by Innovare Academic Sciences Pvt Ltd. This is an open access article under the CC BY license (http://creativecommons. org/licenses/by/4. 0/) DOI: http://dx.doi.org/10.22159/ajpcr.2020.v13i6.37035

\section{INTRODUCTION}

Drug utilization study enables suitable modifications in prescription of pattern to increase the therapeutic benefit and decrease adverse effects [1].

The term arthritis defined as "joint inflammation," but it is referring to conditions that affect the joints and may also affect muscles and other tissues. The most common form of arthritis is degenerative arthritis or osteoarthritis $(\mathrm{OA})$ which is results from the breakdown of the tissue inside the joints. The other form inflammatory arthritis result from swelling in the joints.

The most common form of joint disease is OA and the leading cause of pain in elderly people. The prevalence of knee OA globally was $3.6 \%$, and approximately 251 million people had knee OA in 2010. OA is widely known as the most frequent musculoskeletal disorder, mainly occurring in the elderly with a radiographic prevalence of nearly $70 \%$ in persons over age 65. The incidence of $\mathrm{OA}$ is rising; by increasing epidemics of obesity and aging population [2].

$\mathrm{OA}$ is a progressive and painful chronic disease that affects knee, hand, and hip joints. Pain symptoms associated with OA result in increased physical and walking disability [3,4]. Symptoms such as pain and inflammation become visible in middle age till the age of 55 years occurs equally in both sexes. Pain associated with OA may be periarticular in origin rather than intracapsular. Disease burden is related to pain occurrence, frequently leading to functional disability ranging from slight limitation of movements to severe impairment of normal daily living activities [5].

The management of arthritis is complex and relies on a combination of pharmacological and non-pharmacological approaches for most of patients. Because of pain in osteoarthritis patients, it leads to irrational use of many medication. This misuse leads to intoxication and occurrence of adverse drug reactions, hospitalization, and increase in treatment cost [6].

In the management of $\mathrm{OA}$ to reduce symptoms and functionality or even halt the progression of structural changes and to delay or even avoid the need for prostheses. The management of OA has simple approaches such as weight loss (in obesity), exercise, lifestyle alterations, use of analgesics, and topical agents. Therapeutic measures consist of non-pharmacological (e.g., patient education and physical therapy), pharmacological (e.g., the use of analgesics, nonsteroidal antiinflammatory drugs [NSAIDs]) and symptomatic slow-acting drugs in $\mathrm{OA}$ and ultimately surgical treatments (orthopedic surgery including joint replacement). NSAIDs are also most widely prescribed class of medications worldwide and commonly used over the counter $[7,8]$.

NSAIDs cause serious gastrointestinal ulcer and complications are 4 times higher than non-users and reduce gastrointestinal adverse drug reactions by maximizing local delivery and minimizing systemic toxicity [9].

Drug prescription study was conducted in the outpatient department (OPD) at orthopedic department of tertiary care hospital for evaluating drug prescribed trend and to observe and analyze prescribing pattern of drugs use in OA patient.

\section{METHODS}

The present study was conducted in the department of pharmacology in collaboration with department of orthopedics for a period of 1 year at a tertiary care hospital. All newly diagnosed patients of OA receiving 
treatment attending the OPD of orthopedics for complains of OA were included in the study. During the period, a total 630 patients of $\mathrm{OA}$ were found to be attending orthopedic department OPD. Hence, a of total 630 sample size were selected for study.

A prospective observational study was conducted in a tertiary care hospital for a period February 2017-January 2018 which was reviewed. Data from the patient are recorded in case record form and were analyzed for prescription pattern of drug.

\section{Primary objective}

The primary objective of the study was to tudy the current trend of prescribing patterns of the drugs used in the management of $\mathrm{OA}$ at study site.

\section{Secondary objective}

The secondary objective of the study was to analyze the type of therapymonotherapy or combination therapy.

\section{Inclusion criteria}

The following criteria were included in the study:

- Patients of either sex with age $>50$ years, diagnosed with OA receiving NSAID along with antiulcer drugs at the orthopedic department of tertiary care hospital.

- Patients who are willing to participate in the study.

\section{Exclusion Criteria}

The following criteria were excluded from the study:

- Patients with history of gastrointestinal, renal, and liver disease or any psychiatric illness and with surgical indications for the management of $\mathrm{OA}$ are excluded from the study.

- Patients who are not willing to participate in the study.

- Patients who are not ready to give informed consent.

\section{RESULTS}

The present study was conducted in the department of orthopedics in which patient from OPD was studied for a period of 12 months, in which 630 patients of diagnosed $\mathrm{OA}$ were enrolled. The assessment was done in the age group of $>50$ year of either sex, newly diagnose $\mathrm{OA}$ patients. The present study assesses types of NSAIDS, antiulcer drugs prescription and the type of group of other drugs.

In details of gender distribution of $\mathrm{OA}$, the number of patient of $\mathrm{OA}$ gender-wise distribution in male and female was $45.24 \%$ and $54.76 \%$, respectively (Table 1)

In details of age distribution in $\mathrm{OA}$, age group of 51-57 years, i.e., 243 number of patients (38.57\%), followed by the age group of 58-64 years and 65-71 years of age group with 187 number of patients (29.68\%) and 130 number of patients (20.64\%), respectively (Table 2).

Table 1: Details of gender distribution in OA patients

\begin{tabular}{ll}
\hline Gender distribution & Number of patient (\%) \\
\hline Male & $285(45.24)$ \\
Female & $345(54.76)$ \\
Total & $630(100)$ \\
\hline
\end{tabular}

Table 2: Details of age distribution in OA patients

\begin{tabular}{ll}
\hline Age distribution(years) & Number of patients (\%) \\
\hline $51-57$ & $243(38.57)$ \\
$58-64$ & $187(29.68)$ \\
$65-71$ & $130(20.64)$ \\
$72-78$ & $64(10.16)$ \\
$79-88$ & $6(0.95)$ \\
Total & $630(100)$ \\
\hline
\end{tabular}

Disease distribution shows that sites of OA were knee, hip, hand, and spine among which knee joint was high with disease distribution in 382 number of patients, i.e., $51.34 \%$, followed by hip joint involvement in 151 number of patients, i.e., $20.3 \%$ (Table 3).

In this study, risk factors in OA patients, old age was the most common risk factor, in 251 number of patients, i.e., $39.84 \%$ followed by obesity in 187 number of patients, i.e., $29.68 \%$ (Fig. 1).

Details of drug prescribed in OA patients, most common drug prescribed was diclofenac in 465 number of prescriptions, i.e., $73.8 \%$ followed by the second most common drug prescribed was paracetamol in 439 number of prescriptions, i.e., $69.68 \%$ (Table 4 ).

In class of drug prescribed, NSAIDs were in 1074 number of prescriptions, i.e., $92.19 \%$, followed by corticosteroid in 37 number of prescriptions, i.e., $3.18 \%$ in $\mathrm{OA}$ patients, followed by opioid analgesics in 31 number of prescription, i.e., $2.66 \%$ (Table 5).

In route of drug administration, oral route was in 1165 number of prescriptions, i.e., $87.46 \%$, followed by injectable route in 106 number of patients, i.e., $7.96 \%$, followed by topical route in 61 number of prescriptions, i.e., $4.58 \%$ (Table 6 ).

In antiulcer agents used in OA patients, most common antiulcer agent prescribed in OA patient was ranitidine in 509 number of patients,

Table 3: Details of disease distribution of patients in $\mathrm{OA}$

\begin{tabular}{ll}
\hline Disease distribution & Number of patients (\%) \\
\hline Knee & $382(51.34)$ \\
Hip & $151(20.3)$ \\
Hand & $66(8.87)$ \\
Spine & $145(19.49)$ \\
Total & $744(100)$ \\
\hline
\end{tabular}

Table 4: Details of drug prescribed in osteoarthritis patients

\begin{tabular}{ll}
\hline Drug name & Number of prescriptions (\%) \\
\hline Diclofenac & $465(73.8)$ \\
Paracetamol & $439(69.68)$ \\
Aceclofenac & $30(4.76)$ \\
Ibuprofen & $35(5.55)$ \\
Tramadol & $27(4.28)$ \\
Nimesulide & $22(3.49)$ \\
Naproxen & $2(0.31)$ \\
Piroxicam & $21(3.33)$ \\
Etoricoxib & $12(1.9)$ \\
Valdecoxib & $3(0.47)$ \\
Celecoxib & $3(0.47)$ \\
Rofecoxib & $4(0.63)$ \\
Lornoxicam & $14(2.22)$ \\
Etodolac & $5(0.79)$ \\
Diacerein & $17(2.69)$ \\
Glucosamine & $23(3.65)$ \\
Prednisolone & $29(4.6)$ \\
Indomethacin & $2(0.31)$ \\
Deflazacort & $8(1.26)$ \\
Dextropropoxyphene & $4(0.63)$ \\
\hline
\end{tabular}

Table 5: Distribution based on class of drug prescribed in $\mathrm{OA}$ patients

\begin{tabular}{ll}
\hline Class of drug & Number of prescriptions (\%) \\
\hline NSAIDs & $1074(92.19)$ \\
Corticosteroids & $37(3.18)$ \\
Opioid analgesics & $31(2.66)$ \\
Glucosamine & $23(1.97)$ \\
\hline
\end{tabular}


i.e., $80.79 \%$ followed by omeprazole in 53 number of patients, i.e., $8.53 \%$ followed by pantoprazole followed by rabeprazole, sucralfate, and esomeprazole, respectively (Fig. 2).

The concomitant therapy used in $\mathrm{OA}$ patients shows calcium lactate in 549 number of prescriptions, i.e., $72.42 \%$ followed by multivitamin B-complex in 96 number of prescriptions, i.e., $12.66 \%$ followed by cholecalciferol and muscle relaxants (Table 7).

Combination therapy was most commonly used in 475 number of patients i.e. $75.4 \%$ followed by monotherapy in 155 number of patinets i.e. $24.6 \%$ of Osteoarthritis patients (Table 8).

Combination therapy was used in 475 number of patients, of which 420 number of patients, i.e., $88.42 \%$ were prescribed two drug therapy followed by three drug therapy in 50 number of patients, i.e., $10.53 \%$ followed by more than three drug therapy were used in only $1.0 \%$ of patients (Table 9).

In drug therapy, in $\mathrm{OA}$ patient shows that Diclofenac+Paracetamol most common combination used in 290 number of patients, i.e., 69.54\%, followed by Paracetamol+Ibuprofen in 28 number of patients, i.e., $6.71 \%$ followed by Paracetamol+Aceclofenac in 25 number of patients, i.e., $6 \%$ (Table 10 ).

In details of three drug therapy, most commonly used prescription (i.e., diclofenac+paracetamol+prednisolone) in nine number of patients of OA (Fig. 3).

\section{DISCUSSION}

Arthritis is an acute or chronic inflammation of joint which is accompanied by pain, swelling, and stiffness resulting either from infection or injury. Most common symptom is pain which is associated with poor quality of life. NSAIDs are the treatment of choice for OA for alleviating the pain associated with this condition (Table 11) [10].

In the present study (Table 1), number of patient of OA gender-wise distribution in male and female was $45.24 \%$ and $54.76 \%$, respectively. It correlates with the study conducted by Anjali et al. (2016) where OA was more common in female $91(55.15 \%)$ patients than male patients $74(44.84 \%)[11]$

This study was conducted in patients of $\mathrm{OA}$ of age more than 50 years (Table 2) shows that age-wise distribution of total of 630 patients was with more number of patients in age group of 51-57 years, i.e., 243 number of patients (38.57\%), followed by the age group of 58-64 years and 65-71 years of age with 187 number of patients (29.68\%) and 130 number of patients $(20.64 \%)$, respectively. Hence, this present study was more prevalent in age group of 51-57 years of age compared with Anjali et al. (2016) and Sahayam et al. (2016), which shows OA more prevalent in the age group of 51-65 years of age, i.e., in 74 number of patients (44.84\%), of which 165 patients of $\mathrm{OA}$ and in 54 number of patients $(58.69 \%)$ of which 92 patients of OA, respectively $[11,12]$.

In this study, disease distribution (Table 3 ) shows that sites of $\mathrm{OA}$ were knee, hip, hand, and spine among which knee joint was high with disease distribution in 382 number of patients, i.e., $51.34 \%$, followed by hip joint involvement. Our study compared with Jhanwar et al. (2012) study which shows that disease distribution most common at knee joint in 811 number of patients, i.e., $82.9 \%$ and next common site of disease distribution at hip joint [13].

In the present study (Fig. 1), risk factors in OA patients were old age, obesity, family history, fractures, and others such as gender, ethnic characteristic, sports, and joint deformity. Of which old age was the most common risk factor, in 251 number of patients, i.e., $39.84 \%$ followed by obesity, which was correlates with Gurung et al. (2016) study which shows that old age was most prevalent risk factors in 67 number of patients, i.e., $58.77 \%$ of 114 patients of OA [14]
In this study, details of drug prescribed in OA patients (Table 4), most common drug prescribed was diclofenac in 465 number of prescriptions, i.e., $73.8 \%$ followed by the second most common drug prescribed was paracetamol in 439 number of prescriptions, i.e., 69.68\%, NSAIDs which were most commonly used than other class of drug. Jhanwar et al. (2012) study and Yuganeswaran et al. (2018) showed that most common prescribed drugs were diclofenac and paracetamol in OA patients $[13,15]$.

Table 6: Distribution based on route of administration in $\mathrm{OA}$ patients

\begin{tabular}{ll}
\hline Route & Number of prescriptions $(\mathbf{n = 1 3 3 2}) \mathbf{( \% )}$ \\
\hline Oral & $1165(87.46)$ \\
Injectable & $106(7.96)$ \\
Topical & $61(4.58)$ \\
\hline
\end{tabular}

Table 7: Details of concomitant therapy in $\mathrm{OA}$ patients

\begin{tabular}{ll}
\hline Concomitant therapy & Number of prescriptions (\%) \\
\hline Muscle relaxant & $50(6.59)$ \\
Cholecalciferol & $63(8.31)$ \\
Calcium lactate & $549(72.42)$ \\
Multivitamin B-complex & $96(12.66)$ \\
\hline
\end{tabular}

Table 8: Details of types of therapy in osteoarthritis patients

\begin{tabular}{ll}
\hline Types of therapy & Number of drugs prescribed $(n=630)(\%)$ \\
\hline Monotherapy & $155(24.6)$ \\
Combination therapy & $475(75.4)$ \\
\hline
\end{tabular}

Table 9: Detail approach of combination therapy in OA patients

\begin{tabular}{ll}
\hline Combination therapy & Number of patients (\%) \\
\hline Two drug & $420(88.42)$ \\
three drug & $50(10.53)$ \\
More than three drug & $5(1.05)$ \\
Total & $475(100)$ \\
\hline
\end{tabular}

Table 10: Details of two drug therapy in $0 A$ patients

\begin{tabular}{ll}
\hline Two drug therapy & Number of patients (\%) \\
\hline Diclofenac+Paracetamol & $290(69.54)$ \\
Paracetamol+Aceclofenac & $25(6)$ \\
Paracetamol+Ibuprofen & $28(6.71)$ \\
Paracetamol+Tramadol & $16(3.84)$ \\
Paracetamol+Nimesulide & $12(2.88)$ \\
Diclofenac+Tramadol & $1(0.24)$ \\
Diclofenac+Nimesulide & $2(0.48)$ \\
Piroxicam+Paracetamol & $5(1.2)$ \\
Naproxen+Prednisolone & $1(0.24)$ \\
Piroxicam+Prednisolone & $1(0.24)$ \\
Etoricoxib+Paracetamol & $2(0.48)$ \\
Paracetamol+Prednisolone & $5(1.2)$ \\
Lornoxicam+Diclofenac & $1(0.24)$ \\
Diclofenac+Glucosamine & $4(0.96)$ \\
Diclofenac+Prednisolone & $3(0.72)$ \\
Diclofenac+Deflazacort & $4(0.96)$ \\
Piroxicam+Diclofenac & $1(0.24)$ \\
Etoricoxib+Diclofenac & $1(0.24)$ \\
Valdecoxib+Paracetamol & $1(0.24)$ \\
Rofecoxib+Deflazacort & $1(0.24)$ \\
Lornoxicam+Paracetamol & $6(1.44)$ \\
Etodolac+Paracetamol & $3(0.72)$ \\
Paracetamol+Glucosamine & $2(0.48)$ \\
Paracetamol+Indomethacin & $1(0.24)$ \\
Etodolac+Diclofenac & $1(0.23)$ \\
Total & $417(100)$ \\
\hline
\end{tabular}




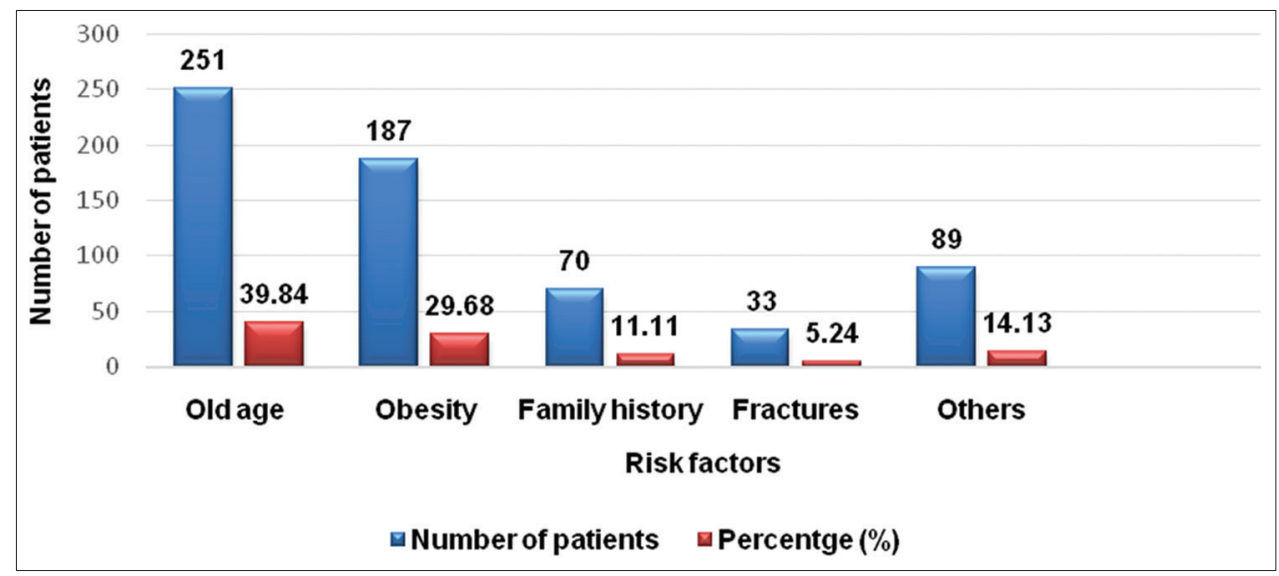

Fig. 1: Details of risk factors in osteoarthritis patients

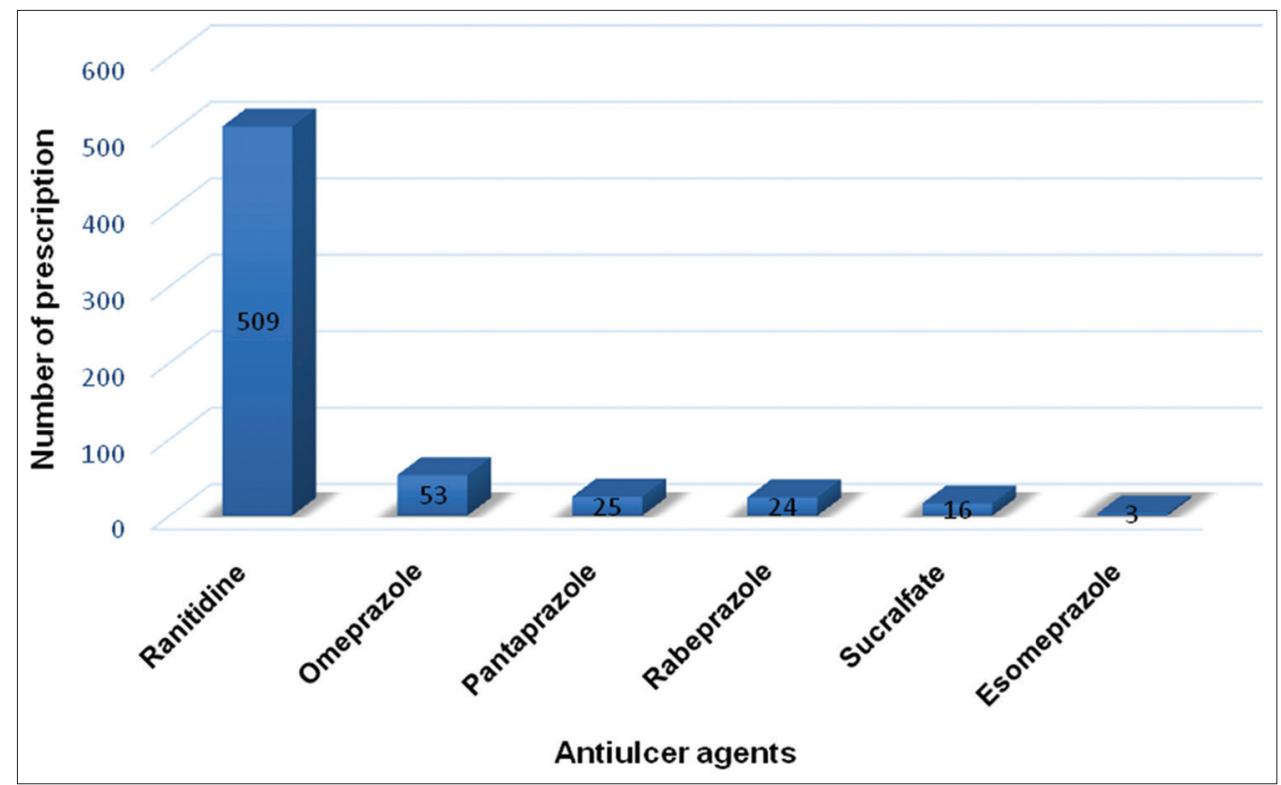

Fig. 2: Distribution of antiulcer agents used in osteoarthritis patients

Table 11: Details of more than three drug therapy in $0 A$ patients

\begin{tabular}{ll}
\hline More than three drugs & $\begin{array}{l}\text { Number } \\
\text { of patients } \\
\text { (\%) }\end{array}$ \\
\hline Diclofenac+Paracetamol+Diacerien+Glucosamine & $2(40)$ \\
Diclofenac+Paracetamol+Tramadol+Indomethacin & $1(20)$ \\
Paracetamol+Ibuprofen+Diacerien+Glucosamine & $1(20)$ \\
Lornoxicam+Paracetamol+Diacerien+Glucosamine & $1(20)$ \\
Total & $5(100)$ \\
\hline
\end{tabular}

In the present study (Table 5), class of drug prescribed was NSAIDs in 1074 number of prescriptions, i.e., $92.19 \%$ followed by corticosteroid in 37 number of prescriptions, i.e., $3.18 \%$ in OA patients. When our study correlate with Sahayam et al. (2016), Purkayastha et al. (2016), and Anjali et al. (2016) study, where the most common prescribed drug class was NSAIDs in $75.1 \%, 81.33 \%$, and $70.4 \%$, respectively, in $\mathrm{OA}$ patients. Hence, NSAIDs are most common class of drug prescribed in OA patients with above study explanation $[10,11,16]$

In our study (Table 6), most commonly used route of drug administration was oral route in 1165 number of prescriptions, i.e., $87.46 \%$ followed by injectable route in 106 number of patients, i.e., 7.96\% followed by topical route in 61 number of prescriptions, i.e., $4.58 \%$ compare with
Anjali et al. (2016) study and Ahmed et al. (2012) study showed that route of drug administration was prescribed by an oral route followed by topical route and injectable in OA patients have similar finding with the present study $[2,11]$.

In this study (Fig. 2), antiulcer agents were also prescribed with each prescription of NSAIDs to counteract the gastrointestinal side effects, most common antiulcer agent prescribed in OA patient was ranitidine in 509 number of patients, i.e., $80.79 \%$ followed by omeprazole in 53 number of patients, i.e., $8.53 \%$ followed by pantoprazole followed by rabeprazole, sucralfate, and esomeprazole which correlate with Gurung et al. (2016) study shows that ranitidine was most common gastroprotective drug prescribed in OA patients with $69.66 \%$ of patients followed by omeprazole, rabeprazole, and pantoprazole drugs were prescribed shows near about same findings as compared with the present study [14].

In this study, the concomitant therapy (Table 7) used in OA patients shows calcium lactate in 549 number of prescriptions, i.e., $72.42 \%$ followed by multivitamin B-complex in 96 number of prescriptions, i.e., $12.66 \%$ followed by cholecalciferol and muscle relaxants. From the above explanation, calcium lactate was most commonly used concomitant therapy in our study for improvement of health condition of OA patients. The study conducted by Gupta et al. (2018), Patil et al. (2016), and Jadhav et al. (2011) shows that calcium supplement was 


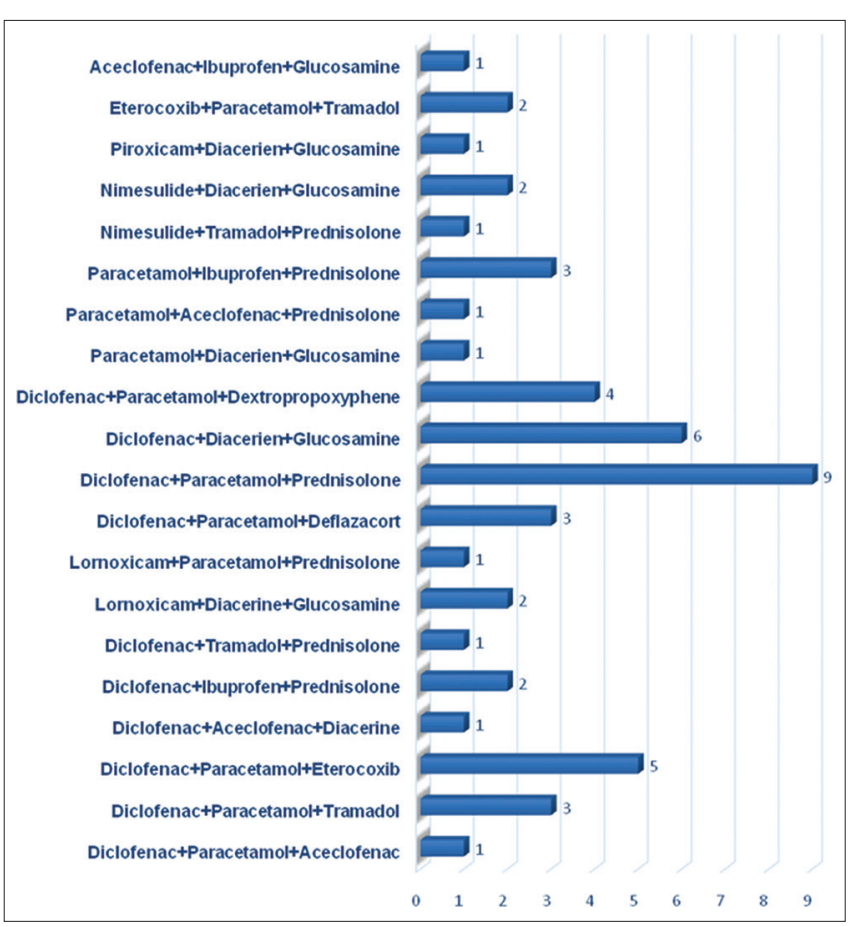

Fig. 3: Details of three drug therapy in osteoarthritis patients

most common concomitant therapy prescribed for OA patients, which have similar finding in accordance with our study [17-19].

In the present study, the details of types of therapy (Table 8) were also shows that combination therapy was most common therapy in 475 number of patients, i.e., $75.4 \%$ followed by monotherapy in 155 number of patients, i.e., $24.6 \%$ of 0 A patients, which was compared with study conducted by Gurung et al. (2016), Yuganeswaran et al. (2018), and Ahmed et al. (2012) shows that combination therapy was most commonly used in $72 \%, 67.02 \%$, and $78.26 \%$, respectively, in OA patients, which was more than monotherapy in accordance with present study and Sahayam et al. (2016) study $[2,10,14,15]$.

Our study gives that details of combination therapy (Table 9) were used in 475 number of patients, of which 420 number of patients, i.e., $88.42 \%$ were prescribed two drug therapy, followed by three drug therapy in 50 number of patients, i.e., $10.53 \%$ followed by more than three drug therapy was used in only $1.0 \%$ of patients. From above discussion, two drug therapy most commonly used in more number of patients than other combination therapy. When this present study correlates with Ahmed et al. (2012), Gurung et al. (2016), and Jhanwar et al. (2012) shows that two drug therapy was most commonly used than other combination therapy $[11,14]$.

In the present study, two drug therapy in OA patient (Table 10) shows that Diclofenac+Paracetamol most common combination used in 290 number of patients, i.e., $69.54 \%$, followed by Paracetamol+Ibuprofen in 28 number of patients, i.e., $6.71 \%$ followed by Paracetamol+Aceclofenac. The study conducted by Ahmed et al. (2012), Gurung et al. (2016), and Yuganeswaran et al. (2018) study shows that Diclofenac+Paracetamol was the most common prescription used in OA patients which show similar findings in accordance with our study ${ }^{[2,14,15]}$.

\section{CONCLUSIONS}

- The principal aim of drug utilization research is to facilitate the rational use of drugs in populations. The rational use of drug implies the prescription of a well-documented drug at an optimal dose. Without knowledge of how drugs is being prescribed and used, it is difficult to initiate a discussion on rational drug use or to suggest measures to improve prescribing habits.
- The WHO suggests that drug utilization studies are needed in every health care setting. Data are useful for preparing essential drug lists and standard treatment protocol.

- In a developing country like India, a National Drug Policy is needed to rationalize the drug use. To achieve this, it is very important to determine drug use pattern and monitor drug use profile over the time and bring it for the awareness among the prescribing doctors.

- The study concludes that the NSAIDs combined with gastroprotective agents were the most appropriate first-line NSAID therapy for many patients. To minimize the occurrence of gastrointestinal toxicity, it is advised to use the National Institute of Clinical Excellence guidance.

- This study shows that OA more common in female patient than male patient.

- Most common disease distribution site was knee in OA patients.

- Old age was the most common risk factor encountered in patients of OA.

- This study shows that in the management of OA, NSAIDs such as diclofenac and paracetamol found to be the most common prescribed drugs.

- Combination therapy prescription most commonly used than single drug therapy prescription. Diclofenac+Paracetamol combination most commonly used.

- In this study, three drug therapy (Diclofenac+Paracetamol +Prednisolone) was prescribed most commonly in OA patients.

- Gastroprotective agents used in every prescription to avoid side effect of NSAIDs in GI system. Most commonly used gastroprotective agent was ranitidine.

- This study gives us idea about current trend of prescription pattern and frequency of drugs used in OA patients.

\section{ACKNOWLEDGMENT}

Author and his team would like to thank to HOD and all staff members in the department orthopedics, tertiary care hospital, Government Medical College, Aurangabad, for their good support and cooperation during the study.

\section{AUTHOR FUNDING}

No funding.

\section{CONFLICTS OF INTEREST}

None declared.

\section{ETHICAL APPROVAL}

The study was approved by the Institutional Ethics Committee.

\section{REFERENCES}

1. Shankar PR, Pai R, Dubey AK, Upadhyay DK. Prescribing patterns in the orthopaedics outpatient department in a teaching hospital in Pokhara, Western Nepal. Kathmandu Univ Med J 2007;5:16-21.

2. Ahmed M, Ali N, Rahman ZU, Khan MM. A study on prescribing patterns in the management of arthritis in the department of orthopaedics. Der Pharm Lett 2012;4:5-27.

3. Rosemann $\mathrm{T}$, Wensing $\mathrm{M}$, Joest $\mathrm{K}$, Backenstrass $\mathrm{M}$, Mahler $\mathrm{C}$, Szecsenyi J. Problems and needs for improving primary care of osteoarthritis patients: The views of patients, general practitioners and practice nurses. BMC Musculoskelet Disord 2006;7:1-9.

4. Nüesch E, Dieppe P, Reichenbach S, Williams S, Iff S, Jüni P. All cause and disease specific mortality in patients with knee or hip osteoarthritis: Population based cohort study. BMJ 2011;342:638.

5. Patel PB, Patel TK. Efficacy and safety of aceclofenac in osteoarthritis: A meta-analysis of randomized controlled trials. Eur J Rheumatol 2017;4:11-8.

6. Petkova VB. Education for arthritis patients: A community pharmacy based pilot project. Pharm Pract (Granada) 2009;7:88-93.

7. Hafez AR, Alenazi AM, Kachanathu SJ, Alroumi AM, Mohamed ES. Knee osteoarthritis: A review of literature. Phys Med Rehabil Int 2014;1:8.

8. Choudhury D, Bezbaruah B. Prescribing pattern of analgesics in orthopedic in-patient department at tertiary care hospital in Guwahati, 
Assam, Northeast India. Indian J Pharmacol 2016;48:377.

9. Manohar VR, Rashmi BR, Chandrashekar R, Souza RD, Rai M, Ullal S. A prospective prescription pattern of non steroidal anti-inflammatory drugs among general practitioners in Dakshina Kannada district of Southern India. Int Res J Pharm 2015;6:420-2.

10. Sahayam JS, Kulandaiammal M, Prakash M. Research article pattern of drug prescribing in osteoarthritis patients attending orthopaedic outpatient department of a tertiary care hospital. J Drug Deliv Ther 2016;6:14-7.

11. Anjali C, Sajeeth CI, Sreeja PA, Abraham BT. A prospective observational study to assess prescription pattern in osteoarthritis and rheumatoid arthritis patients at tertiary care hospital, Palakkad, Kerala. Int J Pharm Pharm Res 2016;6:170-90.

12. Mrsm PA. Prescribing pattern for osteoarthritis in a tertiary care. J Clin Diagn Res 2010;4:2421-6.

13. Jhanwar P, Pandey S, Sharma N, Jhanwar A. Drug utilization study of osteoarthritis in a tertiary care teaching hospital of Rajasthan. Int J Pharm Sci Rev Res 2012;14:35-7.

14. Gurung S, Babu S, Sabu S, Shibu RM, Begum R, Nanjwade BK.
A study on prescribing pattern in the management of osteoarthritis and rheumatoid arthritis in the department of orthopaedics. World J Pharm Pharm Sci 2016;5:1472-93.

15. Santoshkumar RJ, Manjunath S, Sharanabasappa MA. Prescription pattern of anti-malarial drugs in a tertiary care hospital. Asian Pac J Trop Med 2010;3:379-81.

16. Bhaskar R. Prescription pattern of analgesics in orthopaedics OPD at a tertiary care hospital. Int J Basic Clin Pharmacol 2015;5:1.

17. Gupta R, Malhotra A, Malhotra P, Gupta R, Med JR, Mar S. Study of prescription pattern of drugs used in the treatment of osteoarthritis in a tertiary care teaching hospital : An observational study. Int J Res Med Sci 2018;6:985-9.

18. Bai PG, Ravikumar P, Salma U. A study of adverse drug reactions among pulmonary tuberculosis patients treated under dots in a tertiary care hospital. IJBCP Int J Basic Clin Pharmacol 2017;6:779-83.

19. Jadhav P, Mutke A, Sonawane S, Patil B, Naik N, Sonawale A, et al. A prospective observational study to assess quality of life and prescription pattern in osteoarthritis patients at tertiary health centre in Mumbai. Indian J Med Sci 2011;65:58 